\title{
Prevalência de perda auditiva autorrelatada em idosos e fatores associados em Juiz de Fora
}

\author{
Maritza F. B. Caruso, ${ }^{1 *}$ Cláudia Helena C. Mármora, ${ }^{2}$ Francisco E. F. Delgado ${ }^{3}$
}

\begin{abstract}
Resumo
Introdução: A Perda Auditiva (PA) em idosos traz prejuízos funcionais e gastos com saúde pública, mas há poucos estudos no Brasil para verificar sua prevalência e fatores associados. Objetivos: Determinar a prevalência de PA autorrelatada em indivíduos com 65 anos ou mais, moradores da comunidade, $\mathrm{e}$ participantes do estudo sobre fragilidade em idosos brasileiros - FIBRA - JF e investigar os fatores associados. Métodos: Estudo transversal derivado do Estudo Multicêntrico sobre Fragilidade no Idoso Brasileiro - Rede FIBRA - com amostra composta por 427 idosos de Juiz de Fora. Foram considerados sujeitos com PA autorrelatada os idosos que: (1) declararam não ouvir bem em resposta à questão "O(A) senhor(a) ouve bem?"; (2) responderam sim para a questão "O(A) senhor(a) usa aparelho auditivo?". A partir daí estudou-se a associação da PA autorrelatada com variáveis sociodemográficas, clínicas e comportamentais. A análise dos dados foi feita pelo programa SPHINX LEXICA \& EURECA V. 5, estabelecendo a frequência de cada variável, utilizando-se o método de teste do Qui-quadrado $\left(X^{2}\right)$. Resultados: Foi encontrada uma prevalência de 24,4\% de PA autorrelatada. Observou-se forte associação com a variável idade ( $\mathrm{p}<0,05$ ), com média de idade de 77,09 anos para os que relataram não ouvir bem. As demais variáveis analisadas não apresentaram significância. Conclusões: Foram encontradas divergências entre prevalência de PA autorrelatada, provavelmente por se tratar de autorrelato e diferenças metodológicas entre os estudos. A variável idade foi a principal associação estabelecida, necessitando-se de mais estudos populacionais para melhor estabelecer outras associações.
\end{abstract}

Descritores: Perda auditiva; Presbiacusia; Envelhecimento; Fatores de risco; Prevalência.

\section{Abstract}

Prevalence of Self-reported Hearing Loss in the Elderly and its Associated Factors in Juiz de Foras

Introduction: Hearing Loss (HL) in the elderly entails functional impairment and public health costs, however, there are few studies in Brazil to verify its prevalence and associated factors. Objectives: To determine the prevalence of self-reported HL in community elderly aged 65 years and over, part of the Fragility in the Brazilian Elderly study - FIBRA - JF. And to investigate associated factors. Methods: Cross-sectional study derived from the Multicentric Study on Fragility in the Brazilian Elderly - FIBRA Network - with a sample composed of 427 elderly people from Juiz de Fora. Elderly subjects who: (1) answered no to the question "Do you listen well?"; (2) answered yes to the question "Do you wear hearing aids?"
1. Faculdade de Fisioterapia. Universidade Salgado de Oliveira. Juiz de Fora, MG, Brasil.

2. Faculdade de Fisioterapia. Universidade Federal de Juiz de Fora. Juiz de Fora, MG, Brasil.

3. Faculdade de Fisioterapia. Universidade Presidente Antônio Carlos. Juiz de Fora, MG, Brasil.

*Endereço para correspondência:

Rua Barbosa Lima, 322, sala 102

Juiz de Fora, MG, Brasil. CEP: 36010-050.

E-mail:mfbreder@gmail.com

Revista HUPE, Rio de Janeiro, 2018;17(2):35-42

Recebido em 07/05/2018. Aprovado em 11/09/2018.

From this, we studied the association of self-reported HL with socio-demographic, clinical, and behavioral variables. The data analysis was done by SPHINX LEXICA \& EURECA V. 5 program, establishing the frequency of each variable, using the chi-square test method $\left(X^{2}\right)$. Results: A prevalence of $24.4 \%$ of self-reported HL was found. There was a strong association with the age variable $(\mathrm{p}<0.05)$, with the average age for those who did not hear well of 77.09 years. The other variables analyzed did not show significance. Conclusions: Differences were found in the prevalence of self-reported HL, probably because it was a self-report and there were methodological differences between the studies. The age variable was the main association established, requiring more population studies to better establish other associations.

Keywords: Hearing loss; Presbycusis; Aging; Risk factors; Prevalence.

\section{Resumen}

Prevalencia de pérdida auditiva autorreferida en idosos y factores asociados en Juiz de Fora

Introducción: La Pérdida auditiva (PA) en ancianos trae perjuicios funcionales y gastos con la salud pública, pero hay pocos estudios en Brasil para verificar su prevalencia y factores asociados. Objetivo: determinar la prevalencia de PA autorreferida en individuos con 65 años o más, habitantes de la comunidad, y participantes del estudio sobre fragilidad en ancianos brasileños - FIBRA - JF y investigar los factores 
asociados. Métodos: Estudio transversal derivado del Estudio Multicéntrico sobre Fragilidad en el Anciano Brasileño - Red FIBRA - con muestra compuesta por 427 ancianos de Juiz de Fora. Se les consideraba sujetos con personas mayores de auto-reporte de AP que: (1) dijeron que no escucharon bien a la pregunta; "El (la) señor(a) oye bien?" (2) respondió sí a la pregunta "Usted utiliza un audífono?". Partindo de eso se estudió la asociación de la PA autorreferida con variables sociodemográficas, clínicas y comportamentales. El análisis de los datos fue realizado por el programa SPHINX LEXICA \& EURECA V. 5, estableciendo la frecuencia de cada variable, utilizando el método de prueba del Chi-cuadrado

\section{Introdução}

A perda auditiva (PA) em idosos, denominada de presbiacusia quando relacionada do processo natural de envelhecimento, é uma a doença crônica com elevada prevalência, ainda não bem definida, na população brasileira, afetando cerca de $30 \%$ da população com mais de 65 anos. $^{1-3}$

Suas causas incluem alterações fisiológicas e degenerativas em estruturas relacionadas à audição com o aumento da idade e modificações nas áreas corticais cognitivas, que podem não estar envolvidas exclusivamente com a audição., ${ }^{4,5}$ Porém, considerase também a associação com fatores extrínsecos e intrínsecos, geralmente desencadeadores ou potencializadores da PA, como: hereditariedade, fatores metabólicos e vasculares, hipertensão arterial sistêmica (HAS), arteriosclerose, diabetes mellitus (DM) e infecções, além de exposição a ruídos ocupacionais ${ }^{6,7}$ e fatores sociais, também denominados socioacusia, termo criado, segundo Baraky, ${ }^{8}$ para se referir aos fatores sociais predisponentes à $\mathrm{PA}$ do idoso.

Fatores potencializadores incluem: uso de drogas, medicamentos ototóxicos e tabaco; trauma acústico; fatores nutricionais; prática de atividades físicas; estresse; nível de escolaridade e renda; exposição diária a ruído ambiental e utilização de fones de ouvido. ${ }^{7-11}$

A PA acarreta impactos na qualidade de vida dos idosos e de suas famílias, gerando prejuízos biológicos e psicossociais com prejuízos para a conversação, dificuldade em compreender mensagens faladas, tendência ao isolamento social, depressão, distúrbios cognitivos e redução da prática de atividades físicas. ${ }^{10-12}$ Outras consequências menos destacadas são relacionadas à má postura, desequilíbrios, quedas e traumas, geralmente complicadores para a saúde dos idosos. ${ }^{8}$
$\left(X^{2}\right)$. Resultados: Se encontró una prevalencia del $24,4 \%$ de PA autorreferida. Se observó una fuerte asociación con la variable edad ( $\mathrm{p}<0,05$ ), con promedio de edad para los que relataron no oír bien de 77,09 años. Las demás variables analizadas no representaron significancia. Conclusiones: Se encontraron divergencias entre prevalencia de PA autorreferida, probablemente por tratarse de autorreferencia y diferencias metodológicas entre los estudios. La variable edad fue la principal asociación establecida, necesitando más estudios poblacionales para mejor establecer las asociaciones.

Palabras clave: Pérdida auditiva; Presbiacusia; Envejecimiento; Factores de riesgo; Prevalencia.

O custo elevado dos procedimentos de reabilitação auditiva é um dos fatores que levaram o Ministério da Saúde a instituir a Política Nacional de Atenção à Saúde Auditiva em 2004. ${ }^{13}$ Observa-se, assim, um aumento gradual dos estudos de prevalência da PA e fatores associados na população brasileira visando à organização de políticas públicas e estratégias de enfrentamento deste problema, apesar de ainda haver falta de estudos populacionais de âmbito nacional sobre este tema.,114

Um dos desafios para os estudos é a realização da avaliação clínica da audição, que deve utilizar instrumentos para avaliar o funcionamento periférico e central do sistema auditivo, sem deixar de considerar os fenômenos cognitivos que podem estar associados ao declínio da função auditiva. O exame de audiometria é considerado o padrão ouro para o diagnóstico de PA; porém, problemas de orçamento e dificuldades metodológicas limitam sua realização em estudos populacionais. ${ }^{11,15,16}$

Apesar de serem subjetivos e representarem uma PA autorrelatada, que pode não ser fidedignamente condizente com a realidade, estudos por meio de questionários autoavaliativos fornecem pistas sobre a prevalência da PA na população e se traduzem em um meio rápido e econômico para proporcionar estimativas em grandes populações. ${ }^{11,15,17,18}$

OPrograma de Prevenção da Cegueira e da Surdez da OMS se empenha na criação de um banco de dados sobre a deficiência auditiva a partir de uma única pergunta sobre como o indivíduo percebe sua audição.11,19 Observase a realização de estudos de validação de inventários de avaliação da PA autorrelatada e declínio auditivo, ${ }^{20}$ bem como uma tendência a realizar estudos sobre prevalência de PA autorrelatada e verificação de fatores associados 
em banco de dados de estudos populacionais mais amplos que se dispõem a colher grande quantidade de informações. $6,11,14,15,19,21-23$

A partir destes pressupostos este estudo teve como objetivo determinar a prevalência de perda auditiva autorrelatada em indivíduos com 65 anos ou mais, moradores da cidade de Juiz de Fora, MG. Além disso, buscou-se analisar a associação das variáveis sociodemográficas, clínicas e comportamentais com a PA autorrelatada.

\section{Métodos}

Trata-se de uma pesquisa qualiquantitativa, de delineamento transversal, derivada do estudo da Rede FIBRA, polo UERJ. A amostra foi composta por 427 idosos que participaram da pesquisa da Rede FIBRA no município de Juiz de Fora. Este estudo conta com a aprovação do Comitê de Ética e Pesquisa da Universidade Federal de Juiz de Fora - Parecer de aprovação n⿳0 195.631 .

Foram considerados sujeitos com PA autorrelatada todos os idosos que preencheram os seguintes critérios: (1) declararam não ouvir bem em resposta à questão "O(A) senhor(a) ouve bem?"; (2) respondeu sim à questão "O(A) senhor(a) usa aparelho auditivo?". A partir daí estudou-se a associação da PA autorrelatada com as variáveis sociodemográficas, clínicas, comportamentais e funcionais.

Consideraram-se variáveis sociodemográficas as seguintes: sexo, idade, estado civil, etnia, ocupação, alfabetização e renda familiar. Como variáveis clínicas foram elencadas as doenças crônicas autorrelatadas, diagnosticadas por médico (HAS, acidente vascular encefálico, DM e depressão). Como variáveis comportamentais foram selecionadas tabagismo e etilismo.

Para a análise das informações colhidas por meio dos formulários de entrevistas da rede FIBRA, foi construído um banco de dados pela tabulação e codificação dos dados que foram analisados pelo programa SPHINX LEXICA \& EURECA V. 5, estabelecendo a frequência de cada variável na amostra levantada.

Para realizar a montagem dobanco de dados, tornouse necessário proceder às seguintes etapas: determinação de que cada uma das perguntas corresponderia a uma variável; codificação das variáveis; categorização das respostas fechadas; formação do banco de dados e análise estatística descritiva de cada variável.

O método de teste do Qui-quadrado $\left(X^{2}\right)$ foi utilizado por se tratar de um estudo de dispersão de frequência, no qual as variáveis são qualitativas. Ressalta-se que foram utilizadas, como entrada inicial neste estudo, tabelas de contingência (cruzamento) entre a categoria e as variáveis correspondentes, a partir das quais foram calculadas as distâncias entre linhas e colunas compostas por categorias de variáveis qualitativas. Tais distâncias são baseadas na métrica do Qui-Quadrado $\left(X^{2}\right)^{24}$

\section{Resultados}

Foi encontrada uma prevalência de 24,4\%, 104 idosos, que relataram não ouvir bem, enquanto que $75,6 \%$ (323 idosos) relataram que ouvem bem.

Houve uma diferença significativa entre os grupos que relataram ouvir bem e não ouvir bem quanto ao item "usa aparelho auditivo". Em sua maioria, 18 idosos (17,3\%) que relataram usar aparelho auditivo, também relataram não ouvir bem, fator este significativamente relevante, enquanto que somente 2 idosos $(0,6 \%)$ usuários de aparelho auditivo relataram ouvir bem, valor considerado insignificante dentro do banco de dados analisado. Sendo assim, para a verificação das associações da PA autorrelatada com as variáveis sociodemográficas, clínicas e comportamentais, foi utilizada somente a variável "O(a) senhor(a) ouve bem?".

A associação da perda auditiva autorrelatada com as variáveis sociodemográficas, clínicas e comportamentais está descrita nas tabelas 1 e 2 abaixo.

\section{Discussão}

Estudos que apontam a prevalência da PA autorrelatada por idosos apresentam tanto valores afins quanto muito divergentes quando comparados aos do presente estudo.

Malta e colaboradores identificaram uma prevalência de 1,1\% de PA autorrelatada no Brasil, sem diferença entre os sexos, utilizando dados da Pesquisa Nacional de Saúde. ${ }^{14}$ Apesar da amostra não conter somente idosos, a PA tendeu a aumentar nas idades mais elevadas, apresentando significância a partir de 40 anos.

Quevedo e colaboradores fizeram uma análise da prevalência de PA autorrelatada de informantes primários comparada à prevalência informada por informates proxy analisando dados de um inquérito populacional de base domiciliar em Porto Alegre. ${ }^{15}$ Este estudo foi realizado com indivíduos de todas as faixas etárias. Eles identificaram uma prevalência de 22,5\% de PA para os informantes primários, sendo que, na amostra de indivíduos com 60 anos ou mais, representada por 115 casos, a prevalência de PA autorrelatada foi de 35,81\%. 


\section{Artigo original}

Tabela 1. Prevalência de perda auditiva autorrelatada por idosos segundo fatores sociodemográficos

\begin{tabular}{|c|c|c|c|}
\hline & $\mathrm{n}$ & $\%$ & Valor de $\mathrm{p}^{*}$ \\
\hline \multicolumn{4}{|l|}{ Faixa etária (anos) } \\
\hline $65-66$ & 1 & 1,0 & \multirow{3}{*}{$<0,01$} \\
\hline $68-70$ & 4 & 3,8 & \\
\hline$\geq 76$ & 59 & 56,7 & \\
\hline \multicolumn{4}{|l|}{ Sexo } \\
\hline Masculino & 39 & 37,5 & \multirow{2}{*}{0,072} \\
\hline Feminino & 65 & 62,5 & \\
\hline \multicolumn{4}{|l|}{ Cor/etnia } \\
\hline Branca & 80 & 76,9 & \multirow{3}{*}{0,585} \\
\hline Mulata/cabocla/parda & 17 & 16,3 & \\
\hline Preta & 7 & 6,7 & \\
\hline \multicolumn{4}{|l|}{ Estado civil } \\
\hline Casado ou vive com companheiro(a) & 51 & 49 & \multirow{4}{*}{0,641} \\
\hline Viúvo(a) & 39 & 37,5 & \\
\hline Solteiro(a) & 10 & 9,6 & \\
\hline Divorciado/separado(a) & 4 & 3,8 & \\
\hline \multicolumn{4}{|l|}{ Ocupação } \\
\hline \multicolumn{4}{|l|}{ Trabalha atualmente } \\
\hline Sim & 10 & 9,6 & \multirow{2}{*}{0,284} \\
\hline Não & 94 & 90,4 & \\
\hline \multicolumn{4}{|l|}{ Aposentado } \\
\hline Sim & 74 & 71,2 & \multirow{2}{*}{0,132} \\
\hline Não & 30 & 28,8 & \\
\hline \multicolumn{4}{|l|}{ Pensionista } \\
\hline Sim & 38 & 36,5 & \multirow{2}{*}{0,908} \\
\hline Não & 66 & 63,5 & \\
\hline \multicolumn{4}{|l|}{ Alfabetização } \\
\hline Alfabetizado & 96 & 92,3 & \multirow{2}{*}{0,197} \\
\hline Não alfabetizado & 8 & 7,7 & \\
\hline \multicolumn{4}{|l|}{ Nível de escolaridade } \\
\hline Nunca foi à escola & 8 & 7,7 & \multirow{7}{*}{0,197} \\
\hline Curso de alfabetização de adultos & 2 & 1,9 & \\
\hline Primário & 46 & 44,2 & \\
\hline Ginásio & 20 & 19,2 & \\
\hline Científico clássico & 18 & 17,3 & \\
\hline Curso superior & 8 & 7,7 & \\
\hline Pós-graduação & 4 & 0,9 & \\
\hline
\end{tabular}

*Nível de significância $(p<0,05)$ 
Maritza F. B. Caruso e cols. • Perda auditiva autorrelatada em idosos

Tabela 2. Prevalência de perda auditiva autorrelatada por idosos segundo variáveis clínicas e comportamentais

\begin{tabular}{|c|c|c|c|}
\hline & $\mathrm{n}$ & $\%$ & Valor de $\mathrm{p}^{*}$ \\
\hline \multicolumn{4}{|c|}{ Deficiência visual auto-relatada } \\
\hline Sim & 51 & 49 & \multirow{2}{*}{$<0,01$} \\
\hline Não & 53 & 51 & \\
\hline \multicolumn{4}{|c|}{ Hipertensão } \\
\hline Sim & 32 & 30,8 & \multirow{2}{*}{0,083} \\
\hline Não & 72 & 69,2 & \\
\hline \multicolumn{4}{|c|}{ Acidente vascular encefálico } \\
\hline Sim & 2 & 1,9 & \multirow{2}{*}{0,372} \\
\hline Não & 102 & 98,1 & \\
\hline \multicolumn{4}{|c|}{ Diabetes } \\
\hline $\operatorname{Sim}$ & 7 & 6,7 & \multirow{2}{*}{0,145} \\
\hline Não & 97 & 93,3 & \\
\hline \multicolumn{4}{|c|}{ Depressão } \\
\hline Sim & 19 & 18,3 & \multirow{2}{*}{0,212} \\
\hline Não & 85 & 81,7 & \\
\hline
\end{tabular}

Uso de tabaco

Fuma atualmente

$\operatorname{Sim} 6$

Não

97

5,8

0,310

Nunca fumou algum dia

Nunca fumou

Já fumou e largou

58

39

Frequência de consumo de bebidas alcoólicas

Nunca

Uma vez por mês ou menos

2-4 vezes por mês

2-3 vezes por semana

4 ou mais vezes por semana

64

26

3

5

6

Doses de bebida ingeridas normalmente

$\begin{array}{lcr}1-2 & 92 & 88,5 \\ 2-3 & 7 & 6,7 \\ 4-5 & 2 & 1,9 \\ 6-7 & 0 & 0 \\ 8-\text { mais } & 3 & 2,9\end{array}$

*Nível de significância $(p<0,05)$ 


\section{Artigo original}

Já Crispim e Ferreira realizaram um estudo de base populacional na cidade de Manaus que investigou a prevalência de PA autorrelatada e fatores associados em 646 idosos e encontraram uma prevalência de $25,7 \%{ }^{2}$

Cruz e colaboradores analisaram uma amostra de 1.115 idosos participantes do Estudo SABE (Saúde, Bem Estar e Envelhecimento) no município de São Paulo e encontraram 30,4\% de PA autorrelatada. ${ }^{21}$ Paiva e colaboradores utilizaram-se do Inquérito de Saúde do Município de São Paulo - ISA - Capital 2003, e encontraram a prevalência de $11,2 \%$ na população de idosos. ${ }^{11}$ Morettin e colaboradores também realizaram um estudo em São Paulo e avaliaram os fatores relacionados à autopercepção da audição de 2.143 idosos derivados do Estudo SABE, identificando que $33 \%$ da amostra referiu queixas auditivas. ${ }^{19}$

Outro estudo, composto por 1.302 pessoas com idade $\geq 65$ anos, constatou uma prevalência de 6,1\% de PA na amostra analisada. Akeem, Taiwo e Oye analisaram dados do Ibadan Study of Ageing, um estudo de coorte longitudinal para verificação do estado de saúde física e mental, bem como a funcionalidade e incapacidade das pessoas idosas, realizado em regiões de língua iorubá da Nigéria. ${ }^{25}$

Um estudo realizado na periferia urbana e rural da Índia com o objetivo de investigar os problemas de saúde autorrelatados por uma amostra de 407 idosos identificou que 257 idosos (61,5\%) relataram problemas auditivos. ${ }^{26}$

Entende-se que as ambivalências encontradas com relação às prevalências nestes estudos sejam decorrentes do uso de metodologias de análise distintas, com alguns estudos de base populacional compostos por indivíduos de diferentes faixas etárias, enquanto outros eram compostos somente por idosos. ${ }^{14}$ Além disso, não se pode desconsiderar que em todos os estudos existe a limitação da própria avaliação, considerada subjetiva, a partir da coleta de dados da PA a partir do autorrelato. Apesar disso, Quevedo e colaboradores apontam que estudos de base populacional baseados no autorrelato, quando comparados com a audiometria, têm identificado valores de sensibilidade e especificidade aceitáveis. ${ }^{15}$

Além disso, supõe-se também que as dicotomias encontradas possam ser decorrentes da influência de fatores predisponentes para PA. A maioria dos sujeitos da pesquisa da Nigéria era (1.002 - 77,0\%) residente em zona rural ou semiurbana, fator que reduz a presença de ruídos ambientais. Dos 79 entrevistados (6,1\%) que relataram perda de audição, 22 eram residentes em área rural (27,8\%), 41(51,9\%) fumavame $40(50,6 \%)$ relataram etilismo sem associação estatística significante.

Já os sujeitos da pesquisa realizada na Índia eram, em sua maioria, de baixa renda (45,6\%), apresentavam baixo nível de escolaridade $(42,8 \%$ analfabetos e $38,1 \%$ primeiro grau) e faziam uso do tabaco $(58,97 \%)$, fatores associados a maiores prevalências de PA. Estas divergências apontam para a característica multifatorial da PA do idoso, sendo necessário conhecer e discutir melhor tais fatores.

Consideremos, portanto, as variáveis sociodemográficas (tabela 1), clínicas e comportamentais (tabela 2) associadas à PA autorrelatada. Dos idosos que relataram não ouvir bem, 65 (62,5\%) foram do sexo feminino e $30(37,5 \%)$ do sexo masculino, sem diferença significativa entre os sexos. A maioria da amostra foi do sexo feminino, o que certamente pode interferir nos resultados.

Apesar de não apontar para uma diferença significativa, outros estudos têm demonstrado haver maiores prevalências dePA autorrelatada nos indivíduos do sexo masculino. ${ }^{11,21}$ Morettin e colaboradores verificaram que as mulheres autoavaliaram sua audição de maneira mais positiva que os homens. ${ }^{19}$

Segundo Cruz e colaboradores, maiores prevalências de PA no sexo masculino podem ser explicadas por diferenças hormonais e metabólicas entre sexos e pela maior exposição aos ruídos relacionados ao trabalho ao longo da vida. ${ }^{21}$ Devido ao fato de a PA ser uma afecção multifatorial, deveríamos considerar semelhanças ou distinções de sociedade na qual este idoso estava inserido, além de informações relacionadas ao trabalho. As variáveis disponíveis no banco de dados analisado não englobam dados destes idosos na juventude, tornando, portanto, impossível estabelecer tais associações, considerando-se esta uma limitação metodológica para a real compreensão do fenômeno estudado.

Com relação à etnia, 80 (76,9\%) idosos referiram ser brancos, 17 (16,3\%) se enquadraram no item que representava mulatos, caboclos e pardos, enquanto que $7(6,7 \%)$ declararam-se negros, não havendo diferença estatisticamente significativa associada à PA. Malta e colaboradores também não encontraram diferença segundo raça/cor da pele, porém Cruz e colaboradores apontaram maiores prevalências de PA na população branca. ${ }^{6,14}$

Foi verificada forte associação da PA autorrelatada com a variável idade. Os idosos das faixas etárias mais elevadas tiveram maior chance de apresentar PA. Para os indivíduos que relataram ouvir bem a 
média encontrada foi de 73,58 anos, e para os que relataram não ouvir bem a mesma foi de 77,09 anos. Ao compararmos estas médias de idade verificou-se que não há evidências de que as mesmas sejam significativas para $(\mathrm{p}<0,05)$.

No geral, foi observada diferença estatisticamente significativa para os idosos acima de 76 anos. Cruz e colaboradores analisaram a prevalência da PA autorrelatada em seis áreas urbanas do estado de São Paulo. ${ }^{6}$ Os idosos representaram $77,29 \%$ da amostra que referiu ter PA com maiores prevalências quanto maior a idade. Outros pesquisadores também verificaram maior prevalência de PA autorrelatada nas faixas etárias mais avançadas. ${ }^{2,11,14,15,19,21,25}$

Apesar de não caracterizar um estudo de prevalência de PA autorrelatada, Baraky realizou um estudo descritivo populacional seccional e investigou a prevalência da PA no município de Juiz de Fora, MG, por meio de exame audiométrico. ${ }^{8}$ Seus resultados apontaram que, quanto maior a faixa etária, maior a chance de ocorrência de PA. Na amostra analisada os idosos apresentaram prevalência de PA aproximadamente seis vezes superior às outras faixas etárias. Dos 179 idosos de sua amostra, 26,3\% apresentaram PA, valor muito semelhante ao do presente estudo. Inicialmente, considerou-se que esta relação tão próxima fosse um importante fator para confirmar e continuar a estimular os estudos de base populacional a partir do autorrelato; porém, uma análise mais cuidadosa nos remete ao fato de que a semelhança destes dados é uma simples coincidência, visto que os idosos investigados pelos dois estudos não foram os mesmos.

Estudos que comparam os resultados de PA a partir da avaliação feita por questionários de autoavaliação e do exame audiométrico têm apontado que não existe uma relação entre a queixa e a presença real de PA nas populações de idosos investigadas. A maioria não percebe perdas leves, sendo necessário o encaminhamento para avaliações específicas. 18, 27,28

No estudo de Baraky com estratificação da amostra de idosos para análise observou-se que quanto maior a idade, menores são as ocorrências de PA leve e maiores são as ocorrências de perdas moderadas e severas, dado que condiz com os estudos de autorreferência citados. ${ }^{8}$

A relação da PA com a idade é uma associação bastante aceita na literatura., ${ }^{5}$ De acordo com Mattos e Veras, um estudo populacional realizado na Suécia mostrou que no grupo de indivíduos entre 70 e 80 anos de idade, a PA é de aproximadamente 1-2 dB HL/ano e que entre 80 e 90 anos de idade a deterioração ocorre mais lentamente. ${ }^{16}$ Estes mesmos autores encontraram a prevalência da PA na cidade do Rio de Janeiro, que foi de $42,9 \%$ de 238 idosos avaliados com média de idade de 71,8 anos. Foi utilizada a audiometria tonal e encontraram maior porcentagem para o grau leve de perda de audição.

$\mathrm{Na}$ análise da PA autorrelatada por faixas etárias no presente estudo foi verificado que os idosos abaixo de 70 anos raramente relataram ouvir mal, fato estatisticamente significante. A análise estatística dos idosos de 70-76 anos não apresentou diferença entre os indivíduos que relataram ouvir bem e não ouvir bem, fato que pode ser explicado pela autorreferência e pode ser uma possível exemplificação do problema apresentado por alguns autores quando consideram que os estudos de verificação de prevalência de PA a partir de questionários autoavaliativos apresentam baixa sensibilidade e alta especificidade. ${ }^{21}$

As variáveis relacionadas ao estado civil, ocupação, alfabetização, renda familiar, doenças crônicas autorrelatadas diagnosticadas por médico (HAS, acidente vascular encefálico, DM e depressão), tabagismo e etilismo não representaram fatores significantes no banco de dados analisado. Apesar disso, a análise dos valores de odds ratio apontam para uma maior chance de ocorrer a PA nos seguintes casos: sujeitos que trabalham atualmente (odds ratio $=1.48$ ); não estar aposentado (odds ratio $=1,47$ ); ter HAS (odds ratio $=1,52$ ); ter tido um AVE (odds ratio $=1,97$ ); ter DM (odds ratio $=1,85$ ); fumar atualmente (odds ratio $=1,59$ ); e já ter fumado algum dia (odds ratio $=1,51$ ). Segundo Baraky a associação entre presbiacusia e fatores de risco é controversa e ainda não foi bem estabelecida.[8] Em seu estudo encontrou-se relação da PA com renda familiar, nível de escolaridade, DM, HAS, zumbidos e tabagismo, além de outros já relatados.

A não associação dos fatores citados pode ser explicada pela coleta de dados a partir da autorreferência, não havendo forma de confirmá-las fidedignamente, fator este considerado uma limitação metodológica do presente estudo.

\section{Conclusão}

A prevalência de PA autorrelatada em indivíduos com 65 anos ou mais, moradores da comunidade e participantes do estudo de fragilidade em idosos brasileiros - FIBRA-JF, foi de 24,4\%, observando-se poucas similaridades e muitas divergências com outros estudos de prevalência dePA autorrelatada encontrados, 


\section{Artigo original}

que podem estar relacionadas tanto aos métodos empregados para as diferentes pesquisas quanto às diferenças sociodemográficas e comportamentais, visto que a PA do idoso é de origem multifatorial.

A análise da associação da PA autorrelatada com as variáveis sociodemográficas, clínicas e comportamentais mostrou uma maior prevalência deste declínio sensorial com o aumento da idade. As demais variáveis não apresentaram significância estatística.

Espera-se que os dados levantados e discutidos no presente estudo possam servir de incentivo a novas pesquisas relacionadas à compreensão e ao melhor enfrentamento da PA do idoso. Visto ser esta uma afecção com grande prevalência e que gera inúmeros prejuízos, acredita-se que esta também é passível de ser prevenida, haja vista os múltiplos fatores predisponentes que podem ser controlados a partir de estratégias educativas voltadas à população.

\section{Referências}

1. Jardim DS, Maciel FJ, Lemos SMA. Perfil epidemiológico de uma população com deficiência auditiva. Rev CEFAC. 2016;18(3):746757.

2. Crispim KGM, Ferreira AP. Prevalência de deficiência auditiva referida e fatores associados em uma população de idosos da cidade de Manaus: um estudo de base populacional. Rev CEFAC. 2015;17(6):1946-1956.

3. Brasil. Ministério da Saúde. Política Nacional de Saúde da Pessoa Portadora de Deficiência. Brasília, DF, 2008. [recuperado em 30 janeiro 2012]. Disponível em: http://bvsms.saude.gov.br/bvs/publicacoes/politica_nacional_saude_pessoa_deficiencia.pdf

4. Araújo MZ, Dantas MAA, Alves MJ, et al. Presbiacusia: envelhecimento da audição suas causas e consequências através do levantamento da literatura. Anais CIEH. 2015;2(1).

5. Wong PCM, Jin JX, Gunasekera GM, et al. Aging and Cortical Mechanisms of Speech Perception in Noise. Neuropsychologia. 2009;47(3):693-703.

6. Cruz MS, Oliveira LR de, Carandina L, et al. Prevalência de deficiência auditiva autorrelatada e causas atribuídas: um estudo de base populacional. Cad Saúde Pública. 2009;25(5):1123-1131.

7. Carmo LC, Silveira JAM, Marone SAM, et al. Estudo audiológico de uma população idosa brasileira. Rev Bras Otorrinolaringol. 2008;74(3):342-349.

8. Baraky LR. Prevalência de surdez incapacitante no município de Juiz de Fora, Minas Gerais, Brasil. Tese [Doutorado em Ciências]. Universidade de São Paulo; 2011.

9. Cerioli FV, Andrade MS. A visão dos moradores de uma cidade do interior do Paraná sobre o incômodo causado pelo ruído ambiental. Tuiuti: Ciência e cultura. 2010;43:11-21.

10. Meneses C, Mário MP, Marchori LL de M, et al. Prevalência da perda auditiva e fatores associados na população idosa de Londrina, Paraná: estudo preliminar. Rev CEFAC. 2010;12(3):183-09.

11. Paiva KM, Cesar CLG, Alves MCGP, et al. Envelhecimento e deficiência auditiva autorrelatada: um estudo de base populacional.
Cad de Saúde Pública. 2011;27(7):1292-1300

12. Costi BB, Olchik MR, Gonçalves AK, et al. Perda auditiva em idosos: relação entre autorrelato, diagnóstico audiológico e verificação da ocorrência de utilização de aparelhos de amplificação sonora individual. Revista Kairós Gerontologia. 2014;17(2):179192.

13. Ministério da Saúde (Brasi). Portaria n. 2.073/GM, de 28 de setembro de 2004. Institui a Política Nacional de Atenção à Saúde Auditiva. Brasília, 2004. [recuperado em 17 janeiro 2012]. Disponível em: http://dtr2001.saude.gov.br/sas/PORTARIAS/Port2004/ GM/GM-2073.htm

14. Malta DC, Stopa SR, Canuto R, et al. Prevalência autorreferida de deficiência no Brasil, segundo a Pesquisa Nacional de Saúde, 2013. Ciênc Saúde Coletiva. 2016;21(10):3253-3264.

15. Quevedo ALA de, Leotti VB, Goulart BNG de. Análise da prevalência de perda auditiva autodeclarada e fatores associados: informante primário versus proxy. Cad Saúde Pública. 2017;33(6):e0076216. doi: 10.1590/0102-311x00076216.

16. Mattos LC, Veras RC. A prevalência da perda auditiva em uma população de idosos na cidade do Rio de Janeiro: um estudo seccional. Revista Brasileira de Otorrinolaringologia. 2007;73(5):654-659.

17. Kirk KM, McGuire A, Nasveld PE, et al. A.Comparison of self-reported and audiometrically-measured hearing loss in the Australian Defence Force. Int J Audio. 2012;51(4):294-298.

18. Sousa MGC, Russo ICP. Audição e percepção da perda auditiva em idosos. Rev Soc Bras Fonoaudiol. 2009;14(2):241-246.

19. Morettin M, Cardoso MRA, Lebrão ML, et al. Fatores relacionados à autopercepção da audição entre idosos no município de São Paulo - Projeto SABE. Saúde Coletiva. 2008;5:168-172.

20. Deepthi R, Kasthuri A. Validation of the use of self-reported hearing loss and the Hearing Handicap Inventory for elderly among rural Indian elderly population. Arch Gerontol Geriatr. 2012;55(3):762-767.

21. Cruz MS, Lima MCP, Santos JLF, et al. Deficiência auditiva autorrelatada por idosos no Município de São Paulo, Brasil: prevalência e fatores associados (Estudo SABE, 2006). Cad de Saúde Pública. 2012;28(8):1479-1492.

22. Lasisi AO, Abiona T, Gureje O. The prevalence and correlates of self reported hearing impairment in the lbadan study of ageing. Trans R Soc Trop Med Hyg. 2010;104(8):518-523.

23. Almeida SP, Falcão JM. Self-reported hearing-loss in the Portuguese population: evidence from the 4th National Health Survey. Acta Med Por. 2009;22(3):223-232.

24. Greenacre MJ. The carrol-green-shaffer scaling in correspondence analysis: a theoretical and empirical appraisal. J Mark Res. 1989;26:358-365.

25. Akeem OL, Taiwo A, Oye G. The prevalence and correlates of self-reported hearing impairment in the Ibadan Study of Ageing. Trans R Soc Trop Med Hyg. 2010;104(8):518-523.

26. Thakur RP, Banerjee A, Nikumb VB. Health problems among the elderly: A cross-sectional study. Annals of Medical and Health Sciences Research. 2013;3(1):19-25.

27. Samelli AG, Negretti CA, Ueda KS, et al. Comparação entre avaliação audiológica e screening: um estudo sobre presbiacusia. Braz J Otorhinolaryngol. 2011;77(1):70-76.

28. Teixeira AR, Freitas CLR, Millão LF, et al. Relação entre a queixa e a presença de perda auditiva em idosos. Arq Int Otorrinolaringol. 2009;13(1):78-82. 